\title{
Análisis de la rigidez del primer radio en bipedestación con un nuevo dispositivo: estudio exploratorio de viabilidad
}

\author{
Analysis of stiffness of the first ray during weightbearing with a new device: a feasibility \\ exploratory study
}

\author{
Sergio Miralles Ruíz ${ }^{1}$, Víctor Mateos Selma y Javier Pascual Huerta² \\ ${ }^{1}$ Clínica Podológica Sergio Miralles, Castellón, España. ${ }^{2}$ Clínica del Pie Elcano, Bilbao, España
}

Palabras clave:

Primer radio, rigidez, primer metatarsiano, estática, bipedestación, flexión dorsal, medición caracterización, viabilidad, módulo de elasticidad.

\section{Resumen}

Introducción: La medición de la movilidad del primer radio ha generado controversia debido a su valoración subjetiva durante la exploración del pie. Esta ha sido históricamente realizada de forma manual y cuantifica la movilidad del primer radio en flexión dorsal, siendo esta medición un tanto subjetiva.

Pacientes y métodos: El presente estudio cuantifica la rigidez o resistencia a la dorsiflexión del primer radio con un nuevo dispositivo que mide el desplazamiento vertical de dicha estructura (de 0 a $10 \mathrm{~mm}$ ), además de la fuerza en newtons, en bipedestación en sujetos sanos.

Resultados: Se estudiaron un total de 39 pies de 22 pacientes asintomáticos. Se creó una gráfica de rigidez en flexión dorsal para el primer radio, juntando todos los datos de la muestra. La gráfica ofrecía un comportamiento lineal muy similar a lo esperable teóricamente hasta $5.3 \mathrm{~mm}$ de desplazamiento vertical (44 newtons de fuerza). A partir de esta distancia el comportamiento fue más errático y no lineal, posiblemente debido a acomodaciones del pie de los pacientes durante el test.

Conclusiones: Aunque a día de hoy no existe un aparato capaz de medir la resistencia a la dorsiflexión del primer radio en bipedestación, el presente estudio trata de realizar un análisis exploratorio con un nuevo dispositivo para cuantificar dicha medición en sujetos no patológicos, aportando datos que pueden resultar interesantes para futuras investigaciones.

Keywords:

First ray, stiffness, first metatarsal, static, weightbearing, dorsiflexion, measurement, characterization, feasibility, elastic modulus.

\begin{abstract}
Introduction: The mobility of the first ray has generated controversy due to its subjective assessment during exploration of the foot. Historically, this assessment has been done manually for quantification of first ray dorsal excursion being these measurements somewhat subjective.

Patients and methods: The present study quantifies the stiffness or first ray dorsiflexion with a new device that measures the vertical displacement of the metatarsal head from 0 to 10 millimeters, in addition to the vertical force, measured in newtons.

Results: Thirty-nine feet of 22 asymptomatic were tested in the device. By pooling all data, a stiffness graph was created showing the behaviour of first ray stiffness. The graph showed a lineal behaviour which fitted with theoretical predictions from 0 to $5.3 \mathrm{~mm}$ of vertical displacement ( 44 newtons). From that point, the graph showed an erratic and nonlinear behaviour, probably because of foot adaptations of the patients during the test.
\end{abstract}

Conclusions: Although today there is no single device able of measuring the dorsiflexion resistance of the first ray during standing, the present study tries to perform an exploratory and feasibility analysis with a new device in non-pathological subjects. The study gives interesting data on first ray dorsiflexion stiffness behaviour that could be used in future studies. 


\section{INTRODUCCIÓN}

La movilidad del primer radio ha sido clásicamente valorada mediante la prueba clínica descrita por Root y cols. ${ }^{1}$ (Figura 1), siendo una de las pruebas más utilizadas en la actualidad en la exploración del pie. Uno de los problemas asociados a este test clínico radica en la incapacidad de medir de forma fiable el desplazamiento del metatarsiano en flexión dorsal junto con la fuerza realizada para movilizarlo, que puede ser muy diferente de unos pacientes a otros y de unos exploradores a otros. Diversos estudios ${ }^{2-4}$ han valorado la movilidad del primer radio, aunque esta siempre se ha realizado en condiciones de descarga, de forma manual o con dispositivos que miden el desplazamiento dorsal del primer radio. Todas estas exploraciones han demostrado ser poco fiables, subjetivas y además no cuantifican el grado o cantidad de fuerza al que se somete el primer radio durante la exploración.

El término estándar que se utiliza habitualmente en la literatura biomecánica para definir la resistencia de carácter mecánico de una estructura corporal a un movimiento es el de rigide ${ }^{5}$. Este concepto tiene en cuenta dos factores: desplazamiento realizado y fuerza aplicada. Aplicado al primer radio este concepto de rigidez podría ser definido como el cociente entre cantidad de movimiento en flexión dorsal del primer radio y la fuerza realizada en flexión dorsal bajo la cabeza del primer metatarsiano. La combinación de las magnitudes de movimiento provocado junto con la de fuerza realizada permite una descripción más precisa de las características mecá-
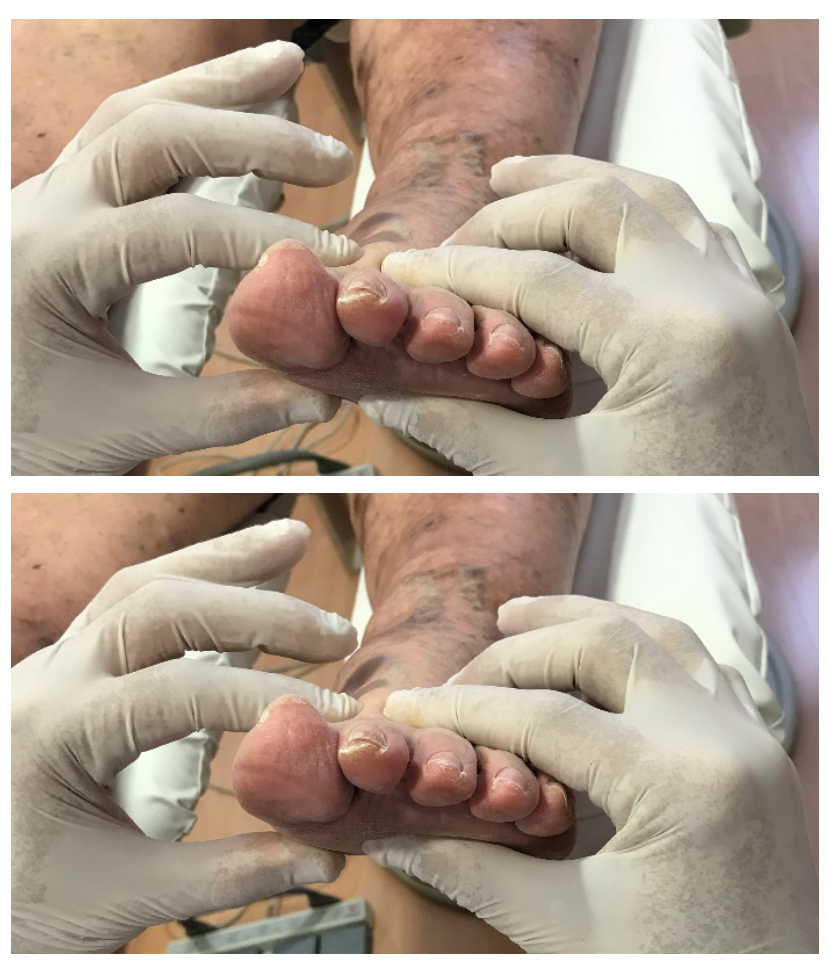

Figura 1. Maniobra de Root para la valoración del movimiento del primer radio. nicas del primer radio que si únicamente nos basamos en el movimiento sin tener en cuenta la cantidad de fuerza exacta ${ }^{6}$.

Tanto el incremento como la disminución de la rigidez o resistencia a la dorsiflexión del primer radio del pie puede acarrear alteraciones que se han relacionado con el desarrollo de múltiples patologías como hallux abductus valgus (HAV), hallux limitus funcional (HLF), hallux rigidus (HR), metatarsalgia de transferencia en los radios menores, aumento de la pronación subtalar, fracturas de estrés en segundo y tercer metatarsianos, sesamoiditis, aumento de la supinación subtalar, etc. ${ }^{7-9}$. Sin embargo, a día de hoy no existe ningún dispositivo que valore la rigidez del primer radio de forma fiable y objetiva y esta valoración se realiza clínicamente de forma subjetiva junto con la experiencia del explorador. Este aspecto es especialmente importante, ya que en base a estas exploraciones se toman decisiones en lo que se refiere tanto a tratamientos quirúrgicos como a tratamientos conservadores del pie. Por todos estos motivos, el presente estudio trata de cuantificar el comportamiento mecánico del primer radio mediante un nuevo dispositivo que mide la resistencia a la dorsiflexión del primer radio en condiciones de carga.

\section{PACIENTES Y MÉTODOS}

Este estudio se clasifica como observacional, descriptivo y transversal. El estudio fue aprobado por el comité ético de la Universidad de Valencia, con código H1513760827611, como parte de una investigación de mayores dimensiones de cuya muestra forman parte los pacientes del presente estudio.

\section{Población de estudio}

Para la realización de este trabajo se estudiaron pacientes que acudieron a la Clínica Podológica Sergio Miralles (CasteIlón de la Plana, España) desde el 21 de junio de 2018 hasta el 27 de junio de 2018. Los sujetos eran pacientes de la clínica con diversos motivos de consulta a los que se les realizó una anamnesis y una exploración en busca de algún criterio de exclusión que no permitiera al individuo participar en dicho estudio.

Los criterios de exclusión fueron: pacientes menores de 20 años, para asegurar que se haya completado la maduración ósea, historia de cirugía previa en el pie, presencia de condiciones neurológicas que disminuyan la sensibilidad en el pie (diabetes mellitus, neuropatías periféricas, etc.) o que afecten a la estabilidad del sujeto (enfermedad de Parkinson, etc.), presencia de deformidad en pie plano o pie cavo diagnosticado mediante exploración clínica, presencia de patología del primer radio o primera articulación metatarsofalángica (HAV o HL/HR), hiperlaxitud ligamentosa y mujeres embarazadas o que crean que pueden estarlo. Todos los par- 

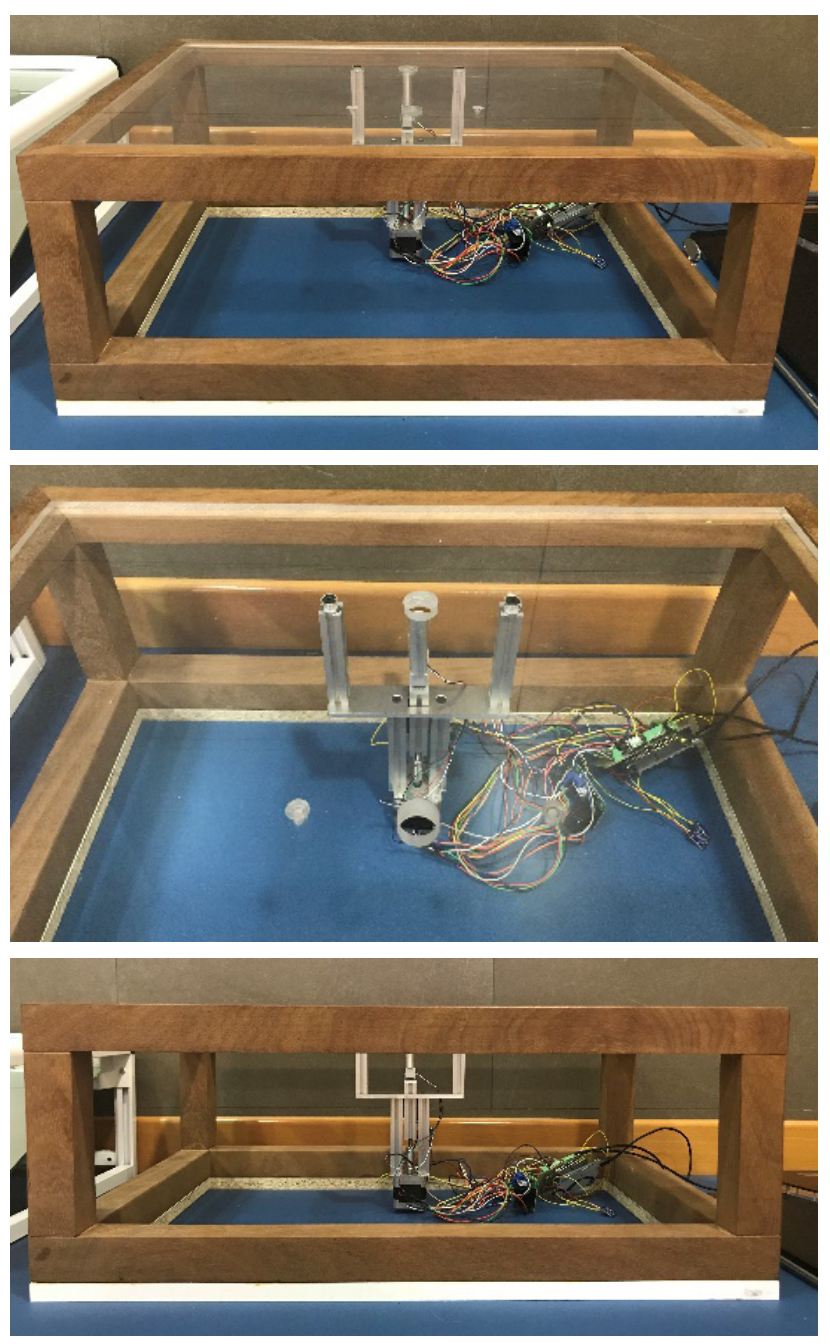

Figura 2. Aparato de medición.

ticipantes firmaron un consentimiento informado para participar en el estudio previo a una explicación de la naturaleza del estudio y de la prueba que se le iba a realizar.

\section{Instrumentación}

El nuevo dispositivo consiste en una plataforma elevada, similar a un podoscopio, donde se apoyan ambos pies, uno de ellos con un orificio bajo la cabeza del primer metatarsiano (Figura 2). Por debajo del orificio se halla un dispositivo con un motor anclado a un vástago central que sube verticalmente empujando la cabeza del primer metatarsiano. El vástago central sube desde $-10 \mathrm{~mm}$ ( $10 \mathrm{~mm}$ bajo la superficie de apoyo) hasta $14 \mathrm{~mm}$ ( $14 \mathrm{~mm}$ por encima de la superficie de apoyo). Cada $0.25 \mathrm{~mm}$ se detiene 3 segundos y mide 20 veces la fuerza que recibe, dando la media de esas 20 mediciones. De esta manera se obtiene la gráfica del coeficiente de rigidez, en la que en el eje de ordenadas (eje Y) tenemos la fuerza realizada por el vástago y en el eje de abscisas (eje X) el desplazamiento vertical del mismo.

El dispositivo está formado por un motor paso a paso, con el propósito de generar la fuerza de empuje vertical del vástago central que tiene un dispositivo de medida en su punta. Los componentes son: una célula de carga resistiva situada en el vástago central, un microcontrolador como elemento de gestión del dispositivo de medida, un microcontrolador como elemento de gestión del motor paso a paso, un microcontrolador como elemento de coordinación de todos los elementos del sistema y como puente entre el dispositivo y el software de control. Este aparato de medida fue construido y testado por un ingeniero mecánico y un informático que participaron en el proyecto.

\section{Protocolo de estudio}

En primer lugar, se realizó una breve anamnesis y una exploración/inspección del pie que permitió conocer si el propio paciente se podía incluir en el estudio teniendo en cuenta los criterios de exclusión. Se anotaron la edad, el peso, la altura y el número de pie de todos los pacientes como datos antropométricos de la muestra, todos ellos referidos por el propio paciente. Con una posición en sedestación o decúbito supino del paciente se realizó una marca exactamente en el lugar donde se pretendía que el vástago incidiera ejerciendo su empuje vertical, coincidiendo con la cabeza del primer metatarsiano en su cara plantar de ambos pies.

A continuación, el sujeto se colocó con ayuda del investigador encima del nuevo dispositivo en bipedestación, con la cabeza del primer metatarsiano sobre el vástago, utilizándose si fuera necesario alguna herramienta (como pudiera ser un pequeño espejo) para comprobar que el vástago coincidía con la marca colocada anteriormente bajo la cabeza del primer metatarsiano. Con las manos el paciente tocaba la pared, sin aumentar la inclinación respecto del punto de apoyo de los pies, para disminuir las oscilaciones propias de la postura bípeda, y se le solicitó que no modificara la posición del pie.

A continuación, a través de un software específico diseñado para el dispositivo, el explorador daba al botón de comienzo de la exploración en la que el vástago central comenzaba a elevarse como se ha comentado anteriormente desde $-10 \mathrm{~mm}$ ( $10 \mathrm{~mm}$ bajo la superficie de apoyo) hasta $14 \mathrm{~mm}$ (14 mm por encima de la superficie de apoyo), deteniéndose durante 3 segundos cada $0.25 \mathrm{~mm}$ de desplazamiento vertical del vástago, obteniendo durante esa pausa 20 mediciones de la fuerza que recibe, proporcionando la media de esas 20 mediciones, si bien es cierto que todos los pacientes no soportaron la subida hasta los $14 \mathrm{~mm}$ por encima del nivel del suelo, por lo que se tuvieron en cuenta los datos hasta los $10 \mathrm{~mm}$ por encima del nivel del suelo. Esta elevación se realizó en todos los sujetos de la muestra y se midieron los resultados obtenidos con dicha elevación, independientemente del rango de 

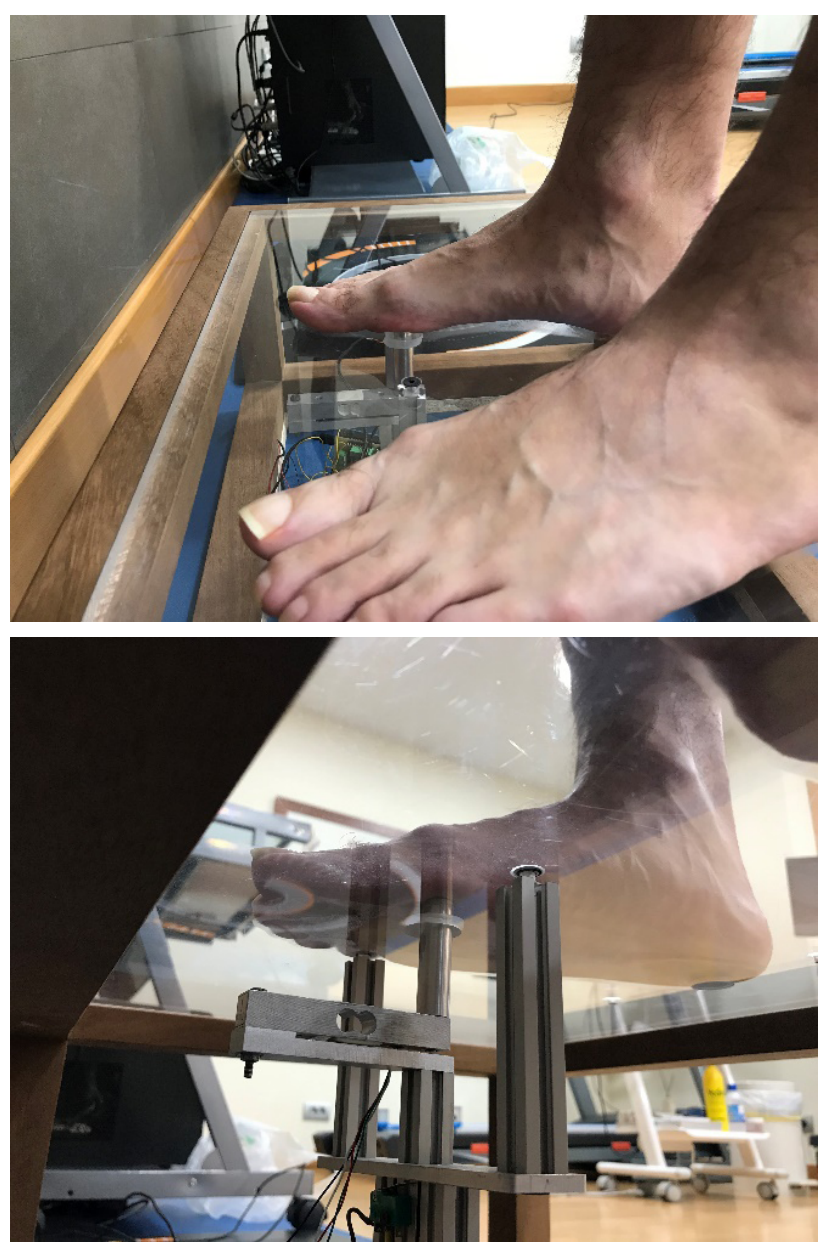

Figura 3. Dispositivo en funcionamiento.

flexión dorsal del primer radio que tuviera el paciente o de las compensaciones que realizó cada paciente. Se solicitó al paciente que procurara no modificar la posición del pie durante la prueba (Figura 3; Vídeo 1).

\section{Análisis estadístico}

El análisis estadístico que se realizó en este estudio se llevó a cabo mediante el programa Microsoft Office, concretamente con la aplicación Excel con su versión del 2013 en la que se obtenía la gráfica final del coeficiente de rigidez. Los valores se presentaron en forma de gráfica en la que en el eje de or- denadas (eje Y) representa la fuerza realizada por el vástago medido en newtons y en el eje de abscisas (eje $X$ ) el desplazamiento del vástago medido en milímetros.

Posteriormente se utilizaron los datos de todos los sujetos para calcular una gráfica media de todos los participantes del estudio junto con un intervalo de confianza al $95 \%$. Se calculó la media y la desviación estándar de los datos antropométricos de la muestra, así como los intervalos de confianza de la gráfica de rigidez con el programa SPSS versión 21 (IBM; Arkmork, EE. UU.).

A su vez, a partir de la gráfica, se calculó el coeficiente de rigidez del primer radio, que es igual a la pendiente de la línea recta de dicha gráfica. Para calcular este coeficiente de rigidez se establecieron de forma visual las zonas de la gráfica que presentaban un comportamiento lineal y sobre esas zonas se calculó el coeficiente como el cociente de la fuerza ejercida por el vástago (en newtons) dividido por la deformación del mismo (en milímetros). La medida utilizada del coeficiente de rigidez fue expresada en newtons/milímetro $(\mathrm{N} / \mathrm{mm})$.

\section{RESULTADOS}

Se estudiaron un total de 39 pies de 22 pacientes que aceptaron colaborar en el estudio y que cumplieron los criterios de inclusión. La Tabla I muestra los datos antropométricos de los sujetos del presente estudio.

La Figura 4 muestra la gráfica de la rigidez del primer radio medida en los 39 pies del estudio. Esta gráfica muestra la relación existente entre fuerza en newtons y el desplazamiento medido por el aparato en milímetros en los 39 pies de los 22 pacientes. La línea central muestra la media de rigidez de todos los sujetos de estudio y las dos líneas superior e inferior muestran el intervalo de confianza al $95 \%$.

Visualmente se establecen 2 partes diferenciadas del comportamiento mecánico del primer radio con el aparato testado. La primera parte muestra un comportamiento que podía ser considerado lineal de la rigidez del primer radio y que va de $0 \mathrm{~mm}$ a hasta algo más de $5 \mathrm{~mm}$ de desplazamiento vertical. La segunda parte va desde $5.4 \mathrm{~mm}$ a $10 \mathrm{~mm}$ de desplazamiento vertical. Esta segunda parte muestra un comportamiento mucho más errático y que difícilmente puede catalogarse como de lineal.

En la primera parte de la gráfica (de 0 a $5.25 \mathrm{~mm}$ de desplazamiento vertical) se pueden observar igualmente 2 segmentos diferenciados. Inicialmente, el comportamiento es de rigidez aumentada de manera progresiva hasta los primeros

Tabla I. Datos antropométricos de los pacientes.

\begin{tabular}{|c|c|c|c|c|c|c|}
\hline Sexo & $\begin{array}{l}\text { Edad } \\
\text { (años) }\end{array}$ & $\begin{array}{l}\text { Altura } \\
(\mathrm{cm})\end{array}$ & $\begin{array}{l}\text { Peso } \\
(\mathrm{kg})\end{array}$ & Talla pie & $\begin{array}{c}\text { N. }{ }^{\circ} \text { pies } \\
\text { Dcho./Izq. }\end{array}$ & $\begin{array}{c}\text { \% Pacientes estudio } \\
\text { unilat./bilat. }\end{array}$ \\
\hline $\begin{array}{l}\text { Hombres } 40 \%(n=9) \\
\text { Mujeres } 60 \%(n=13)\end{array}$ & $44.22 \pm 16.48$ & $167.81 \pm 7.95$ & $68.63 \pm 14.10$ & $40.59 \pm 2.92$ & $20 \mathrm{PD} / 19 \mathrm{PI}$ & $\begin{array}{l}22.7 \% \text { unilateral }(n=5) \\
77.3 \% \text { bilateral }(n=17)\end{array}$ \\
\hline
\end{tabular}




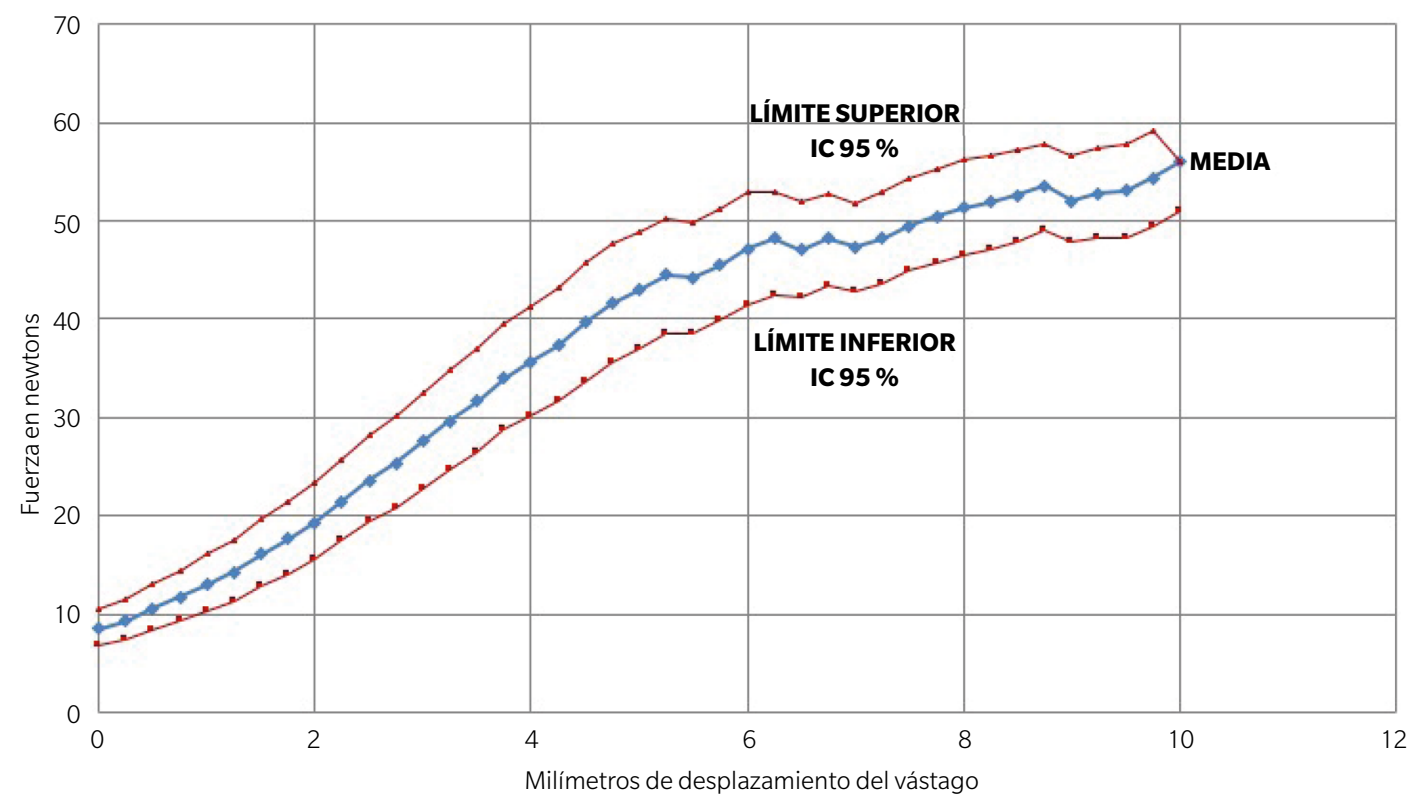

Figura 4. Gráfica de la rigidez del primer radio.

$2 \mathrm{~mm}$ (20 newtons de fuerza), continuando posteriormente con un incremento de forma lineal hasta $5.25 \mathrm{~mm}$ de desplazamiento vertical (44 newtons de fuerza). El coeficiente de rigidez calculado para el primer segmento (de 0 a $2 \mathrm{~mm}$ ) fue de $5.45 \mathrm{~N} / \mathrm{mm}$ y el coeficiente de rigidez calculado para el segundo segmento (de 2 a $5.25 \mathrm{~mm}$ ) fue de $7.87 \mathrm{~N} / \mathrm{mm}$.

En la segunda parte, entre los $5.25 \mathrm{~mm}$ y los $7 \mathrm{~mm}$ de desplazamiento vertical del vástago, se aprecia que los datos que no siguen un patrón lineal como el anterior mostrando una pendiente más suave, es decir, a mayor desplazamiento del vástago la fuerza no aumenta de forma lineal, con alguna variante en negativo, lo cual indica una pérdida de rigidez del primer radio a partir de los 5.3-5.5 mm de desplazamiento. A su vez, entre los $7 \mathrm{~mm}$ y los $8.7 \mathrm{~mm}$, se vuelve a estimar una conducta proporcional por un periodo más corto con una pendiente menos pronunciada, pero de comportamiento más lineal que la anterior. En la última parte, entre los $8.7 \mathrm{~mm}$ y los $10 \mathrm{~mm}$, se contemplan ciertos valores negativos seguidos de una pendiente análoga respecto de la tercera parte de la gráfica. Debido a la pérdida de comportamiento lineal general en esta segunda parte, no se pudo calcular el módulo de elasticidad en este segmento de la gráfica.

\section{DISCUSIÓN}

A día de hoy no existe un aparato capaz de medir la resistencia a la dorsiflexión del primer radio ("rigidez") en bipedestación. El presente estudio trató de realizar un análisis exploratorio con un nuevo dispositivo para caracterizar la rigidez del primer radio en una muestra de sujetos no patológicos. El estudio se realizó de forma muy generalizada, puesto que la edad de los participantes era muy diversa, tanto que el rango de estos estaba entre los 20 y los 71 años. También es cierto que las características antropométricas y las actividades que realizan son muy dispares. Estos aspectos podrían influir directa o indirectamente sobre la resistencia a la dorsiflexión del primer radio, pero no se han tenido en cuenta a la hora de llevar a cabo la investigación.

Las descripciones teóricas ${ }^{10,11}$ y los estudios cadavéricos previamente realizados ${ }^{12}$ sobre el comportamiento del primer radio predicen un incremento gradual de forma no lineal al comienzo del test (primer 15-20\%) cuando las estructuras blandas comienzan a tensarse, de tal forma que el primer radio aumenta su rigidez de forma exponencial. Posteriormente a esta primera fase inicial la predicción es de un comportamiento de crecimiento de la rigidez lineal durante la etapa media de la gráfica (comportamiento elástico) hasta que, en la etapa final, el primer radio aumenta exponencialmente su rigidez debido a un aumento de tensión de las partes blandas que mantienen la estabilidad del primer radio, produciendo un aumento de la fuerza muy grande con pocos milímetros de desplazamiento vertical. Esto ocurre en la fase final en la que las estructuras blandas plantares que estabilizan el primer radio se encuentran en máxima tensión.

Teniendo en cuenta la Figura 4, los datos obtenidos en el presente estudio concuerdan con la descripción en la primera y la segunda fase. En la primera fase encontrada en nuestro estudio existe un incremento gradual de la rigidez que corresponde con el desplazamiento lineal del vástago de 0 a $2 \mathrm{~mm}$ (coeficiente de rigidez de $5.45 \mathrm{~N} / \mathrm{mm}$ ) y que equivale a un intervalo de fuerza de 9 a 20 newtons sobre la primera cabeza metatarsal. Posteriormente existe un aumento lineal de la rigidez del primer radio que se extiende hasta 
aproximadamente la mitad de la gráfica ( $5.25 \mathrm{~mm}$ de desplazamiento vertical del vástago que equivale a 44 newtons) y que permite calcular el coeficiente de rigidez del primer radio durante dicha fase, siendo de $7.87 \mathrm{~N} / \mathrm{mm}$. A partir de aquí el comportamiento de la rigidez medido en los sujetos es más errático y no sigue un comportamiento lineal produciendo, de hecho, una disminución de la rigidez cuando los modelos teóricos predicen un aumento de rigidez a partir de este momento. Este hallazgo puede ser debido a un error en la colocación del pie del paciente en el punto donde el vástago del dispositivo ejerce la elevación vertical o la modificación de la posición del pie que el propio paciente realiza de forma sutil (ej. en forma de supinación del pie o contracción muscular) con objeto de evitar un aumento de las fuerzas del vástago sobre la primera cabeza metatarsal que puede comenzar a sufrir un estímulo doloroso en dicha zona. Es la opinión de los autores del presente trabajo que el cambio sutil en la posición del pie (de forma semiconsciente o inconsciente ante un estímulo que comienza a ser doloroso bajo la primera cabeza metatarsal) es el principal motivo de los hallazgos encontrados en la fase final de la gráfica de la rigidez del primer radio encontrada en el presente estudio. A pesar de que el estudio se explicó a los pacientes que no modificaran la posición de su pie durante el test y el observador vigiló que no se produjeran cambios en la posición del pie por parte del paciente, pequeñas contracciones en la musculatura del pie (ej: tibial posterior, flexor propio del Hallux...) u oscilaciones en la posición bípeda podrían ser responsables de dicho comportamiento.

La rigidez es una medida de la resistencia a las deformidades que sufre un material elástico. Dicho de otro modo, el término "rigidez" describe la fuerza necesaria para realizar una determinada deformación en una estructura. En términos generales, la definición de rigidez como ${ }^{13}$ "'Rigidez' = 'Carga' dividido por 'Deformación'” incluye el concepto de "Deformación" creada por la cantidad de "Carga" soportada. Existe un número enorme de posibles configuraciones del concepto de "Carga" que actúa sobre una estructura (fuerza, momento, estrés, grupos arbitrarios de fuerzas, etc.) unido al concepto de deformación de dicha estructura que puede ser cuantificado (desplazamiento, deformación, ángulo, radio, curvatura, etc.). Por lo tanto, el término "Rigidez" de una estructura requiere siempre una descripción exacta de la configuración de carga y del tipo exacto de deformidad medida. En el presente estudio, el coeficiente de rigidez calculado hace referencia a la fuerza en newtons medida por el vástago sobre la primera cabeza metatarsal dividido por el desplazamiento en milímetros del vástago. Este aspecto es especialmente importante a la hora de comparar los resultados del presente estudio con los realizados en otros estudios. Sobre la gráfica de rigidez como la realizada en el presente estudio, el coeficiente de rigidez calculado es igual a la pendiente de la línea recta en la primera mitad de la gráfica.

El coeficiente de rigidez calculado en el presente estudio es el análogo al módulo de elasticidad o módulo de Young calculado para los materiales elásticos. El módulo de elasticidad se define como el radio de tensión/deformación y se considera como una característica fundamental de ese material. El valor de este módulo elástico generalmente se mide en gigapascales, o GPa, y representa la rigidez del material. Se pueden observar las características mecánicas y estructurales del cuerpo humano en diversos materiales orgánicos. Así, el módulo elástico para un tendón es 0.4 GPa, para la piel $0.5 \mathrm{GPa}$ y para hueso cortical es de $17 \mathrm{GPa}^{14}$. La principal diferencia con el coeficiente de rigidez calculado en el presente estudio consiste en que el concepto de "Carga" calculado en el módulo de Young hace referencia a "Tensión" o "Estrés" del material expresado como la fuerza soportada por unidad de superficie del material, valor que no pudo ser calculado en el presente estudio, por lo que los valores obtenidos en el presente estudio no son comparables con los del módulo de Young estudiados en otros materiales orgánicos. A su vez, es importante entender que el coeficiente de rigidez calculado en el presente estudio hace referencia a la rigidez de un conjunto funcional de segmentos óseos unidos por articulaciones (primer radio) y no de un único material aislado (hueso, ligamento, etc.). Entendemos que el valor obtenido en el presente estudio es una primera aproximación al coeficiente de rigidez del primer radio que debería seguir siendo investigado en posteriores estudios con poblaciones mejor definidas y menos heterogéneas.

El presente estudio presenta ciertas limitaciones que deben de ser tenidas en cuenta a la hora de interpretar los datos. La primera limitación ya comentada es la heterogenicidad de la muestra en cuanto a edades, sexo y datos antropométricos, lo cual puede influir en los resultados obtenidos. Esta heterogenicidad también es aplicable a la posición del pie de los pacientes de la muestra (en pronación o supinación), que al ser un estudio exploratorio de viabilidad no se tuvo en cuenta en los criterios de exclusión y que podría influir en los valores obtenidos de medición de la rigidez del primer radio. Por otro lado, las dimensiones anatómicas de este segmento no son las mismas en todos los pacientes, pero el vástago sí que es el mismo para todos, por esta razón puede existir algún caso en el que el vástago no ejerciera la elevación vertical donde debiera. Igualmente, es cierto que el empuje vertical no es puramente sobre el segmento óseo del primer radio, ya que más plantar a este se encuentra tejido blando como la grasa plantar, aspecto variable entre los pacientes, y el aparato glenosesamoideo. Este es un aspecto importante que debería ser contemplado en posteriores estudios.

En conclusión, el presente estudio aporta datos sobre un nuevo dispositivo para medir la rigidez del primer radio en condiciones de carga estática. Los datos obtenidos muestran concordancia con las predicciones teóricas hasta una carga de 44 newtons (5.25 mm de desplazamiento vertical). A partir de estos valores, los datos muestran un comportamiento más errático que podrían no ser fiables y que podría venir explicado por acomodaciones en la posición del pie del paciente ante la presencia de carga excesiva recibida en el 
primer metatarsiano. Aunque los datos son prometedores, la relevancia de este estudio está en saber que este nuevo dispositivo ofrece datos que parecen ser fiables de resistencia a la dorsiflexión del primer radio en bipedestación hasta $5.25 \mathrm{~mm}$ de desplazamiento vertical del vástago y no más allá de ello. Son necesarios más estudios posteriores para determinar valores de normalidad, valores de rigidez en diferentes grupos con alteraciones en el pie (pie plano, pie cavo, HAV, metatarsalgia mecánica, etc.) y determinar el efecto de la rigidez del primer radio de diferentes actuaciones terapéuticas como tratamientos quirúrgicos, tratamientos con férulas o plantillas y tratamientos de rehabilitación.

\section{DECLARACIÓN DE CONFLICTO DE INTERESES}

Los autores reportan ningún conflicto de interés en relación al presente estudio.

\section{FINANCIACIÓN}

Ninguna.

\section{BIBLIOGRAFÍA}

1. Root ML, Orien WP, Weed JH. Normal and Abnormal Function of the Foot. Vol. 2. Los Angeles: Clinical Biomechanics Corp; 1977.
2. Glasoe WM, Grebing BR, Beck S, Coughlin MJ, Saltzman CL. A Comparison of Device Measures of Dorsal First Ray Mobility. Foot Ankle Int. 2005;26(11):957-61. DOI: 10.1177/107110070502601111.

3. Klaue K, Hansen MD, Masquelet AC. Clinical, Quantitative Assessment of First Tarsometatarsal Mobility in the Sagital Plane and Its Relation to Hallux Valgus Deformity. Foot Ankle Int. 1994;15(1):9-13. DOI: 10.1177/107110079401500103.

4. Glasoe WM, Yack HJ, Saltzman CL. Measuring First Ray Mobility With a New Device. Arch Phys Med Rehabil. 1999;80(1):122-4. DOI. 10.1016/ s0003-9993(99)90320-9.

5. McGraw-Hill Dictionary of Scientific \& Technical Terms, 6E. S.v. "stiffness coefficient." RetrievedSeptember 212019 from https://encyclopedia2. thefreedictionary.com/stiffness+coefficient

6. Kirby KA, Roukis TS. Precise naming aids dorsiflexion stiffness diagnosis. Biomechanics. 2005;July:55-63.

7. Phillips RD, Law EA, Ward ED. Functional Motion of the Medial Column Joints of theFoot during Propulsion. J Am Podiatr Med Assoc. 1996;86(10):474-86. DOI: 10.7547/87507315-86-10-474.

8. Munuera PV, Polo J, Rebollo J. Lenght of First Metatarsal and Hallux in Hallux Valgus in Initial Stage. Int Orthop. 2008;32(4):489-95. DOI: 10.1007/s00264-007-0350-9.

9. Grady JF, Axe TM, Zager EJ, Sheldon LA. A Retrospective Analysis of 772 Patiens with Hallux Limitus. J Am PodiatrMedAssoc. 2002;92(2):102-8. DOI: 10.7547/87507315-92-2-102.

10. Kirby KA, Roukis TS. Precise naming aids dorsiflexion stiffness diagnosis. Biomechanics. 2005;12(7):55-62.

11. Kirby A. Biomecánica del Pie y la Extremidad Inferior II. Arizona: Precision Intracast, Inc.; 2002.

12. Fauth AR, Hamel AJ, Sharkey NA. In vitro measurements of first and second tarsometatarsal joint stiffness. J ApplBiomech. 2004;20(1):14-4.

13. Baumgart F. Stiffness - an unkown world of mechanical science? Injury, Int J Care Injured. 2000;31S:SB14-24. DOI: 10.1016/S00201383(00)80040-6.

14. Özkaya N, Golsdheyder D, Nordin M. Fundamentals of Biomechanics. $4^{\text {th }}$ ed. New York: Springer; 2017. 